\title{
INTELIGENCIA EMOCIONAL, ADICCIÓN AL SMARTPHONE Y MALES- TAR PSICOLÓGICO COMO PREDICTORES DE LA NOMOFOBIA EN ADOLESCENTES
}

\author{
Neryelith Díaz Miranda, Natalio Extremera Pacheco ${ }^{1}$ \\ Universidad de Málaga
}

\begin{abstract}
Resumen
El objetivo de la presente investigación es analizar la relación entre las variables adicción al smartphone, inteligencia emocional, y malestar psicológico con los niveles de nomofobia, así como examinar el gradolos niveles de dichas variables para predecir varianza explicativa de esta fobia comportamental hacia los smartphones. Se pasaron aplicaron diferentes escalas validadas para evaluar inteligencia emocional, adicción al smartphone, malestar psicológico y nomofobia en una muestra de 312 adolescentes de entre 14 y 20 años, de Málaga e Ibiza. Los resultados indicaron que existen unos niveles moderados de nomofobia entre los adolescentes. Los análisis de regresión demostraron que dos dimensiones de la inteligencia emocional (percepción interpersonal y uso de las emociones), la ansiedad y la adicción al smartphone explicaban significativamente varianza de los niveles de nomofobia. Nuestros hallazgos sugieren que los futuros programas relacionados con la prevención de la nomofobia, deberían poner su atención a los niveles de habilidades emocionales, salud mental positiva y estrategias preventivas de uso razonable y no problemático del smartphone.
\end{abstract}

\section{Palabras clave.}

Nomofobia; inteligencia emocional; adicción al smartphone; depresión; ansiedad; estrés.

\section{Introducción}

En la actualidad, los dispositivos móviles, particularmente los teléfonos inteligentes o smartphones, son un objeto fundamental en el desarrollo y funcionamiento diario de las personas, ya que no solo sirven para realizar las tareas iniciales para las que fueron concebidos (p.e. llamar y enviar mensajes de texto), sino que también permiten el acceso y la conexión constante a internet y a la gran red de servicios que en ella se ofrecen. Según las últimas encuestas del Instituto Nacional de Estadística (INE) de 2018 , el $69,8 \%$ de la población adolescente de entre 10 y 15 años dispone de smartphones, encontrándose que, a mayor edad, mayor tendencia a disponer de teléfonos móviles, especialmente a partir de los 13 años.

Recibido: 3 de febrero 2020

Aceptado: 19 de marzo 2020

Correspondencia: Neryerith Díaz Miranda

Email: neryelith@hotmail.com

\begin{abstract}
The objetive of the present research is to analyze the relationship between smartphone addiction, emotional intelligence, psychological distress and levels of nomophobia, as well as the predictive levels of these variables to explain variance in this behavioral addiction towards smartphone. Different instruments was used to assess emotional intelligence, smartphone addiction, psychological distress and nomophobia in a sample of 312 adolescents between 14 and 20 years old, from Malaga and Ibiza. The results showed a moderate levels of nomophobia among adolescentes. The regression analysis showed that two dimensions of emotional intelligence (appraisal of others' emotions and use of emotions), anxiety and smartphone addiction explained significantly variance of nomophobia levels. Our findins suggest that further prevention programs for nomophobia we should pay more attention in fosterior emotional intelligence abilities, positive mental health and teaching preventive strategies related with the reasonable and non-problematic use of smartphone.
\end{abstract}

\section{Key words:}

Nomophobia; emotional intelligence; smartphone addiction; depression; anxiety; stress.

Adicionalmente, un estudio desarrollado por la Fundación Telefónica (2016), señala que el 82,8\% de los teléfonos móviles que hay en España son smartphones. Así mismo, indica que el smartphone es el dispositivo que más utilizan las personas para conectarse a Internet. Las nuevas tecnologías de la información y la comunicación (TIC) se han convertido en una parte esencial de nuestras vidas (Lee, Tam y Chie, 2014; Yildirim y Correia, 2015). La mayoría de las personas, especialmente la población joven, utilizan las nuevas tecnologías para cubrir diferentes tipos de necesidades, como pueden ser estudiar, sentir seguridad, desarrollar el ocio, mantener el contacto con los amigos y muchas otras actividades (Cho y Lee, 2016; Kang y Jung, 2014). Las TIC y los nuevos dispositivos móviles, especialmente los smartphones, están teniendo un fuerte impacto no solo en el cambio de nuestro modo de vida, en el acceso a la información y la manera de comunicarnos, sino también en el surgimiento de nuevas fobias y problemas mentales relacionados con el uso de dichos dispositivos (Bragazzi y Del Puente, 2014; González, León, Pérez y Calvete, 2017), entre ellas la nomofobia.

\section{Nomofobia}

El término nomofobia, resultante de la abreviatura en inglés de "nomobile-phonephobia", fue definido por Bragazzi y Del Puente (2014) 
como "un trastorno de la contemporánea sociedad digital y virtual y hace referencia a las molestias, ansiedad, nerviosismo y angustia causada por no estar en contacto con un teléfono móvil" (p. 156). Este término, fue acuñado por la Oficina de Correos de Reino Unido en 2008, gracias a un estudio comisionado por YouGov, el cual tenía como objetivo evaluar la posibilidad de sufrir trastornos de ansiedad provocados por el uso del teléfono móvil (Bhattachary, Bashar, Srivastava, Singh, 2019). En dicha investigación, se encontró que el 53\% de los participantes mostraban niveles elevados de aprehensión cuando perdían su teléfono móvil, se quedaban sin batería, sin datos móviles o sin cobertura (Yildirim y Correia, 2014). Posteriormente, SecurEnvoy (2012), una compañía de seguridad de Reino Unido, señaló que un $66 \%$ de los 1.000 participantes sufría de nomofobia.

Uno de los primeros estudios sobre la nomofobia, desarrollado por King, Valença y Nardi (2010), señala la nomofobia como un fenómeno que "denota incomodidad o ansiedad al no poder tener contacto con el teléfono móvil u ordenador. Es el miedo a verse tecnológicamente incomunicado, lejos del teléfono móvil o no conectado a la Web" (King et al., 2010, p. 52). En un estudio más reciente, King, Valença, Silva, Sancassiani, Machado y Nardi (2014), definen la nomofobia como: "el miedo moderno a no poder comunicarse a través del teléfono móvil o Internet. [...] La nomofobia es un término que hace referencia a un conjunto de comportamientos o síntomas relacionados con el uso del teléfono móvil. La nomofobia es una fobia situacional relacionada con la agorafobia (p. 28)". En esta última definición, se pone el énfasis sobre el miedo a no poder hacer uso del teléfono móvil y de Internet, así mismo, un punto fundamental, es la expresión de la nomofobia como una fobia comportamental y situacional asociada al miedo a no disponer del teléfono móvil.

Bajo este orden de ideas, en la presente investigación, la nomofobia será entendida en relación a los teléfonos móviles, específicamente a los smartphones. Estos aparatos tecnológicos, gracias a sus múltiples capacidades, facilitan la comunicación instantánea, ayudan a las personas a estar conectadas sin importar el lugar ni la hora y proporcionan constante acceso a la información; es por cuestiones como esas, que las personas se vuelven cada vez más dependientes a sus móviles (Park, Kim, Shon y Shim 2013), lo que agrava los sentimientos de ansiedad causados por la pérdida de contacto con el smartphone.

Atendiendo a Bragazzi y Del Puente (2014), la nomofobia es un trastorno de ansiedad específico que comporta diferentes conductas, entre las cuales se puede encontrar la obsesión por tener uno o más dispositivos adicionales, llevar siempre un cargador consigo, sentir ansiedad y nerviosismo frente al pensamiento de perder el móvil o no poder usarlo por alguna razón, evitar situaciones donde no pueda usar su smartphone, verificar constantemente si tiene notificaciones, dormir junto al teléfono móvil y preferir comunicarse a través de las nuevas tecnologías y no en conversaciones cara-a-cara. Así mismo, existe comorbilidad entre la nomofobia y otros trastornos psiquiátricos, tales como la depresión, el abuso de substancias tóxicas, adicciones comportamentales (adicción a Internet, adicción al smartphone) y, además, aparece asociado a otro tipo de fobias tales como la fobia social (Bragazzi y Del Puente, 2014).

Las fobias situacionales como la nomofobia suponen uno de los cinco tipos de fobias específicas reconocidas en la cuarta edición de Diagnostic and Statistical Manual of Mental Disorders (DSM-IV) e incluidas también en la quinta edición del manual (Bragazzi y Del Puente, 2014). De acuerdo con Choy, Fyer y Lipsitz, "la fobia especifica se caracteriza por un miedo excesivo, irracional hacia una situación u objeto específicos, los cuales son evitados a toda costa o sobrellevados con gran angustia" (2007, p. 267). Por tanto, se experimentan cuando una situación concreta (no poder estar en contacto con el smartphone) evoca un miedo agudo e irracional, el cual conlleva una intensa reacción que puede ser de carácter físico y emocional (Yildirim, 2014).

Aunque la aparición de este fenómeno fóbico es reciente, diversas investi- gaciones están intentando esclarecer las causas y detonantes que inician, mantienen y desarrolla la nomofobia. Entre estas variables, tres dimensiones parecen tener vínculos teóricos y empíricos, a saber, la adicción al smartphone, los déficits en inteligencia emocional y el malestar emocional.

Con respecto a la adicción o uso problemático del smartphone se categoriza como una adicción comportamental (Van Deursen, Bolle, Hegner, y Kommers, 2015); entendida como la incapacidad de limitar el uso del smartphone pese a sus efectos dañinos en el funcionamiento del usuario (Zhitomirsky y, Blau, 2016). Así mismo, se considera que la adicción al smartphone es precursora de los niveles de nomofobia (Y1ld1z, 2018), por lo que, desde esta perspectiva, se plantea que los adolescentes que presentan mayores niveles de adicción al smartphone, son más propensos a sentir miedo de estar alejado de su dispositivo o mostrar más preocupación por no poder tener acceso a él.

En cuanto a la inteligencia emocional, éste es un constructo psicológico que hace referencia a la capacidad del procesamiento de la información emocional, a través de la habilidad de percibir, facilitar, comprender y regular las emociones propias y de los demás (Mayer y Salovey, 1997) En la actualidad no hemos encontrados investigaciones que relacionan los déficits en inteligencia emocional con los niveles de nomofobia, aunque la literatura ha mostrado evidencias empíricas preliminares de las conexiones entre déficits emocionales y la adicción al smartphone (Sun, Liu., y Yu, 2019). Además, se ha encontrado que ciertos rasgos como la inestabilidad emocional (neuroticismo) o extraversión (Amichai-Hamburger, Wainapel y Fox, 2002; Park y Lee, 2013), el estrés (Chiu, 2014; Lee, Chang, Lin y Cheng, 2014; Samaha y Hawi, 2016; Sapacz, Rockman, y Clark, 2016) y la compensación o ganancia emocional (Jeong, Kim, Yum, y Hwang, 2016; Salehan y Negahban, 2013) se asocian significativamente con la adicción al smartphone. Igualmente, algunos trabajos señalan que los adolescentes intentan escapar de sentimientos de soledad, conflictos de su vida social, o de problemas irresolubles a través de la conexión a internet, accediendo desde sus smartphones (Byun et al., 2009) o que los adolescentes con baja inteligencia emocional prefieren las interacciones sociales y comunicaciones online más que cara a cara (Casale, Tella, y Fioravanti, 2013).

Además, aunque no existen aún evidencias sobre las relaciones empíricas entre inteligencia emocional y la nomofobia, la inteligencia emocional ha mostrado ya su vinculación con otras fobias situacionales, tal como la fobia social, considerado uno de los cinco tipos de fobia específica a los que pertenece también la nomofobia. Por ejemplo, trabajos previos han hallado asociaciones significativas entre bajos niveles de inteligencia emocional y mayores puntuaciones en fobia social (Summerfeldt, Kloosterman, Antony, McCabe y Parker, 2010). Así mismo, Jacobs et al. (2008) informaron de vínculos significativos y negativos entre altos niveles de inteligencia emocional y menores síntomas de ansiedad en pacientes con fobia social. En suma, parece que los déficits emocionales podrían estar relacionados con miedos intensos, irracionales y específicos tales como aquellos de carácter tecnológicos propios de la nomofobia.

Por último, los problemas de malestar emocional y salud mental se encuentran asociados con diferentes tipologías y niveles de fobias, subrayándose una alta comorbilidad entre desajustes psicológicos y fobia (Ohayon y Schatzberg, 2010; Wersebe et al., 2018),. aAsí, adolescentes con niveles altos de desajustes psicológicos, muestran fobias $\mathrm{u}$ otros problemas de salud mental que afectan negativamente su desarrollo (Essau, Conradt y Petermann, 1999; Torre y Essau, 2019). Respecto con las fobias tecnológicas, se ha encontrado que las personas que comprueban numerosas veces sus smartphones a lo largo del día, experimentan mayores niveles estrés y ansiedad (Thomée, Härenstam y Hagberg, 2011). De igual forma, Hong et al. (2012), aseguran que ser 
una persona con altos índices de estrés, aumenta los riesgos de ser adicto al teléfono móvil. Por último, otros estudios han relacioando la adicción al smartphone con síntomas de depresión, ansiedad y fatiga mental (Cheever, Rosen, Carrier y Chavez, 2014; Chotpitayasunondh y Douglas, 2016). En general, estos resultados parecen evidenciar la relación existente entre síntomas de malestar emocional y nomofobia.

Por lo anteriormente expuesto y, ya que la literatura recomienda que se realicen más investigaciones estudiando diferentes aspectos de la nomofobia, poniendo especial énfasis en aspectos psicológicos y sociales (Argumosa, Boada y Vigil, 2017; Sezer y Atilgan, 2018; Yildirim y Correia, 2015; Yildiz, 2018), el presente estudio examina los niveles de nomofobia en esta muestra con la principal intención de analizar sus relaciones con las dimensiones de adicción al smartphone, inteligencia emocional, y malestar emocional (i.e. depresión, ansiedad y estrés), asimismo se examina la capacidad predictiva de dichas dimensiones para explicar varianza adicional de los niveles de nomofobia en una muestra de adolescentes. Para ello, se formulan las siguientes hipótesis:

De acuerdo con la literatura previa, se esperan relaciones positivas entre la adicción al smartphone, depresión, ansiedad, estrés y los niveles de nomofobia. Igualmente, se hipotetiza que se encontrarán relaciones negativas entre inteligencia emocional y los niveles de nomofobia. Por último, se espera que las variables adicción al smartphone, inteligencia emocional, y los síntomas de malestar emocional sean variables explicativas y significativas de los niveles de nomofobia.

\section{Método}

\section{Participantes}

Un total de 314 estudiantes procedentes de dos institutos de Málaga capital y provincia ( $72 \%$ ) y otro de Ibiza (28\%), completaron los cuestionarios solicitados. No obstante, la muestra final se compone de 312 participantes, debido a que 2 cuestionarios no fueron cumplimentados correctamente, de los cuales un 46,8\% eran varones y un 53,2\% eran mujeres, con edades comprendidas entre los 14 y 20 años $(M=15,56$, $\mathrm{SD}=1,193)$. Los alumnos se encontraban escolarizados en $3^{\circ}$ de ESO $(41,4 \%), 4^{\circ}$ de $\operatorname{ESO}(31,8 \%), 1^{\circ}$ de bachillerato $(16,6 \%)$ y $2^{\circ}$ de bachillerato $(10,2 \%)$.

\section{Instrumentos}

Junto con las variables socio-demográficas, los participantes cumplimentaron cuatro cuestionarios:

La nomofobia fue evaluada a través del instrumento Nomophobia Questionnaire (NMP-Q) de Yildrim y Correia (2015), adaptado al español por González, León, Pérez y Calvete (2017). Este cuestionario cuenta con 20 ítems, los cuales son respondidos a través de una escala Likert del 1 al 7, donde 1 es totalmente en desacuerdo y 7 es totalmente de acuerdo. El rango de puntuaciones varía de 20 a 140 puntos, indicando a mayor puntuación, mayores niveles de nomofobia. Así mismo, cuenta con cuatro factores, el factor 1 atiende al "no poder comunicarse" (p.ej. "Me inquietaría por no poder comunicarme al momento con mi familia y/o amigos); el factor 2 hace referencia a "la pérdida de conexión" (p.ej. "Me pondría nervioso/a por estar desconectado/a de mi identidad virtual."); el factor 3 estudia el "no ser capaz de acceder a la información" (p.ej. "Me molestaría si no pudiera utilizar mi smartphone y/o sus aplicaciones cuando quisiera."); por último, el factor 4 analiza el "renunciar a la comodidad que otorga el smartphone" (p.ej. "Me daría miedo si mi smartphone se quedase sin batería."). La suma de estas subdimensiones proporciona una puntuación total de los niveles de nomofobia $(\alpha=.94)$

La inteligencia emocional se evaluó mediante la escala Wong and Law Emotional Intelligence Scale (WLEIS; Wong y Law, 2002), adaptada al castellano por Extremera, Rey y Sánchez (2019). Esta escala, com- puesta por 16 ítems, analiza cuatro dimensiones de la inteligencia emocional: percepción intrapersonal (p. ej. "La mayoría de las veces sé distinguir porqué tengo ciertos sentimientos.”), percepción interpersonal (p. ej.

"Conozco siempre las emociones de mis amigos/as a través de sus comportamientos."), uso de las emociones (p. ej. "Siempre me animo a mí mismo para hacerlo lo mejor que pueda.") y regulación de las emociones (p. ej. "Soy capaz de controlar mi temperamento y manejar las dificultades de manera racional.”). Los ítems son respondidos a través de una escala Likert de 7 puntos, donde 1 es completamente en desacuerdo y 7 completamente de acuerdo. La suma de las cuatro dimensiones permite una puntación global de la Inteligencia emocional $(\alpha=.89)$.

Los niveles de malestar emocional fueron evaluados con la Depresion, Anxiety and Stress Scales (DASS-21), de Lovibond y Lovibond (1995), adaptada al español por Bados, Solanas y Andrés (2005). Los participantes responden a 21 ítems en una escala Likert de 4 puntos la frecuencia con la que han experimentado cada situación la semana pasada, donde 0 es "no me pasó" y 3 "me pasó mucho o la mayor parte de tiempo". El DASS-21 supone un conjunto de tres subescalas de 7 ítems, dirigidas a evaluar los diferentes estados emocionales de depresión (p. ej. "No podía sentir ningún sentimiento positivo."), ansiedad (p. ej. "Estaba preocupado/a por situaciones en las cuales podía tener pánico o en las que podría hacer el ridículo") y estrés (p. ej. "Me ha costado mucho descargar la tensión."). La puntuación total del instrumento es satisfactoria $(\alpha=.94)$.

La adicción al smartphone se evaluó utilizando la escala Smartphone Addiction Scale (SAS-SV) de Kwon, Lee, Won, Park, Min y Kim (2013), adaptada al español por López (2015). Cuenta con un total de 10 ítems, a los que se responde con una escala Likert de 1 a 6 , siendo 1 completamente en desacuerdo y 6 completamente de acuerdo. Esta escala, analiza seis sintomatologías causadas por la adicción al smartphone: la pérdida de control (p. ej. "Debido al uso del teléfono móvil he perdido tareas/actividades/ trabajos/etc. previamente planificados.”); interrupción de la familia o escolarización (p. ej. "La gente de mi alrededor me dice que uso demasiado mi teléfono móvil.”); ignorar las consecuencias físicas o psicológicas (p. ej. "Debido al uso del teléfono móvil he sentido dolor en alguna de mis muñecas o detrás del cuello (por ejemplo, en la nuca), etc.”); preocupación (p. ej "Tengo mi teléfono móvil en mente incluso cuando no lo uso."); tolerancia (p. ej. "Uso mi teléfono móvil más de lo que había previsto inicialmente.") y retracción/retirarse (p. ej. "Me siento impaciente e inquieto/a cuando no tengo mi teléfono móvil."). Las puntuaciones totales pueden ir de 10 a 60 , donde a mayor puntuación mayor adicción al smartphone $(\alpha=.84)$.

\section{Procedimiento}

Se llevó a cabo un diseño con muestreo intencional de carácter no probabilístico para la recogida de datos. El estudio presenta un diseño de investigación transversal, donde las variables a analizar fueron obtenidas mediante cuestionarios autoadministrados, cumplimentados durante los meses de diciembre de 2018 y abril de 2019, en las ciudades de Málaga e Ibiza. Dichos institutos dieron su autorización expresa y el consentimiento para la pasación de los cuestionarios. Los participantes fueron informados del carácter anónimo y confidencial de los datos, participando de manera voluntaria en la investigación

\section{Análisis estadístico}

Todos los análisis estadísticos fueron llevados a cabo utilizando el IBM SPSS versión 25. Previo a los análisis principales, se realizaron cálculos preliminares para verificar la distribución normal, determinar los casos extremos, así como para comprobar los supuestos de multicolinealidad y homocedasticidad.

En primer lugar, se llevó a cabo un análisis descriptivo de las puntuaciones de nomofobia. A continuación, se realizaron análisis de correlación entre las variables, utilizando al coeficiente de correlación de Pearson. Y, por último, se llevó a cabo un análisis de regresión lineal múltiple jerárquico 
para comprobar el grado explicativo de las variables independientes (inteligencia emocional; depresión, ansiedad, estrés y adicción al smartphone) sobre la variable dependiente (nomofobia).

\section{Resultados}

Los resultados muestran que la prevalencia de la nomofobia en los participantes se encuentra en un nivel moderado $(X=67.87, D T=25.03)$, basándonos en la clasificación dada por Yildirim y Correia (2015). De acuerdo a estos criterios, una puntuación de 20 indicaría la ausencia de nomofobia, puntuaciones entre 20 y 60 corresponderían a un nivel leve de nomofobia, resultados entre 60 y 100 señalarían un nivel moderado de nomofobia y, por último, puntuaciones mayores de 100 harían referencia a una nomofobia severa. Por otro lado, examinando los factores de la escala, se aprecia como "el miedo a no poder comunicarse" $(X=3.89, D T=1.55)$ es el factor que más puntúa, seguido de "no ser capaz de acceder a la información" $(X=3.44$, $D T=1.43)$ y de "renunciar a la comodidad que otorga el smartphone" $(X=3.20, D T=1.44)$, el factor que menos puntúa ha sido el de "la pérdida de conexión" $(X=2.95, D T=1.39)$.

Tabla 1.

Estadisticos descriptivos de Nomofobia y sus factores

\begin{tabular}{lcccc} 
& Min & Max & $X$ & DT \\
\hline Nomofobia & & & & \\
No poder comunicarse & 1 & 7 & 3.89 & 1.55 \\
Perder la conexión & 1 & 7 & 2.95 & 1.39 \\
No ser capaz de acceder a la información & 1 & 7 & 3.44 & 1.43 \\
Renunciar a la comodidad que otorga el smartphone & 1 & 7 & 3.20 & 1.44 \\
Puntuación total & 20 & 130 & 67.87 & 25.03 \\
\hline
\end{tabular}

\section{Análisis de correlaciones bivariadas de Pearson}

Tal como se aprecia en la tabla 2, los análisis de correlación de Pearson mostraron que las dimensiones de uso y regulación emocional mostraron relaciones positivas y significativas con nomofobia, de modo que a menores puntuaciones en uso de las emociones y en regulación de las emociones, mayores eran puntuaciones en nomofobia. No obstante, la inteligencia emocional total no mostró asociaciones significativas con nomofobia, sugiriendo la necesidad de examinar las relaciones entre las dimensiones específicas de IE y la nomofobia

Respecto a la relación entre las dimensiones de malestar emocional (estrés, ansiedad, depresión) y la nomofobia, se observaron relaciones significativas y positivas, sugiriendo que altos mayores niveles de estrés, ansiedad y depresión se asocian a niveles más altos de nomofobia.

Tabla 2.

Correlaciones entre las variables analizadas

\begin{tabular}{|c|c|c|c|c|c|c|c|c|c|c|}
\hline & 1 & 2 & 3 & 4 & 5 & 6 & 7 & 8 & 9 & 10 \\
\hline 1.Total Nomofobia & 1 & & & & & & & & & \\
\hline $\begin{array}{l}\text { 2.Total Inteligencia } \\
\text { emocional }\end{array}$ & -.110 & 1 & & & & & & & & \\
\hline $\begin{array}{c}\text { 3.Percepción } \\
\text { intrapersonal }\end{array}$ & -.104 & $.868^{* *}$ & 1 & & & & & & & \\
\hline $\begin{array}{c}\text { 4.Percepción } \\
\text { interpersonal }\end{array}$ & .096 & $.721^{* *}$ & $.517^{* *}$ & 1 & & & & & & \\
\hline $\begin{array}{l}\text { 5.Uso de las } \\
\text { mociones }\end{array}$ & $-.175 * *$ & $.838^{* *}$ & $.641^{* *}$ & $.461^{* *}$ & 1 & & & & & \\
\hline $\begin{array}{l}\text { 6.Regulación de } \\
\text { las emociones }\end{array}$ & $-.177 * *$ & $.814^{* *}$ & $.666^{* z}$ & $.344^{* 8}$ & $.624^{* *}$ & 1 & & & & \\
\hline 7.Estrés & $-214^{* *}$ & $-.188^{* *}$ & $-.234^{* *}$ & -.003 & $-.114^{*}$ & $-259 * *$ & 1 & & & \\
\hline 8.Ansiedad & $.254^{* *}$ & $-.168^{* *}$ & $-.237^{* *}$ & $-.144^{*}$ & $-.182^{* *}$ & $-304^{* *}$ & $.740 * *$ & 1 & & \\
\hline 9.Depresión & $.219^{\mathrm{s}}$ & $-246^{* *}$ & $-260=8$ & .006 & $-.268=8$ & $-279=8$ & $.768^{* 8}$ & $.755^{\mathrm{s}}$ & 1 & \\
\hline $\begin{array}{l}\text { 10.Adicción al } \\
\text { smartphone }\end{array}$ & $.556^{ \pm *}$ & $-.140^{*}$ & $-.126^{*}$ & .038 & $-.175^{* *}$ & $-194^{* *}$ & $.197 * *$ & $.174^{* *}$ & $.183^{* *}$ & 1 \\
\hline$x$ & 67.87 & 4.40 & 4.43 & 4.77 & 4.29 & 4.10 & .90 & .78 & .99 & 2.72 \\
\hline DT & 25.03 & 1.03 & 1.24 & 1.31 & 1.24 & 1.31 & .82 & .69 & .74 & 1.02 \\
\hline
\end{tabular}

Nota :**p $<.01 * \mathrm{p}<.05$ X: Media ; DT: Desviación típica
Por último, la adicción al smartphone y la nomofobia, se hallaron relaciones positivas muy significativas entre ambos conceptos; de esta forma, mayores puntuaciones en adicción al smartphone se asociaban a mayores niveles informados de nomofobia por los adolescentes.

\section{Análisis de regresión jerárquico}

Diversos análisis de regresión lineal jerárquico se llevaron a cabo para examinar el papel explicativo de las dimensiones de inteligencia emocional, depresión, ansiedad, estrés y adicción al smartphone sobre los niveles de nomofobia de los adolescentes. En estos análisis, sexo y edad fueron incluidas como covariables.

En la tabla 3 se observa se observa que el modelo total era significativo y explicaba en su conjunto un $34,7 \%$ del total de la varianza de la nomofobia $(F=17.5, p<.01)$. En concreto, un $8,3 \%$ fue explicado por dos dimensiones de inteligencia emocional (percepción interpersonal y uso de las emociones); un 4,6\% fue explicado por los niveles de ansiedad $\mathrm{y}$, finalmente, la adicción al smartphone explicaba un $22,4 \%$ de la varianza en nomofobia, siendo el mayor predictor de esta fobia específica al smartphone.

Tabla 3.

Análisis de regresión jerárquico

\begin{tabular}{llcc}
\hline & $\beta$ & $\mathbf{R}^{2}$ & $\mathbf{\Delta R}^{2}$ \\
\hline Modelo 1 & & $.347^{* *}$ & \\
$\quad$ Sexo & & & \\
Edad & -.025 & & .014 \\
Modelo 2 & -.079 & & \\
Percepción intrapersonal & & & \\
Percepción interpersonal & -.020 & \\
$\quad$ Uso de las emociones & $.186^{* *}$ & $.083^{* *}$ \\
$\quad$ Regulación de las emociones & $-.134^{*}$ & \\
Modelo 3 & .00 & \\
$\quad$ Estrés & & \\
Ansiedad & .010 & \\
$\quad$ Depresión & $.195^{*}$ & \\
Modelo 4 & -.057 & \\
$\quad$ Adicción al smartphone & & \\
\hline
\end{tabular}

Nota : ** $<.01 * \mathrm{p}<.05$

\section{Discusión}

El objetivo principal de la presente investigación fue analizar la relación existente entre las variables adicción al smartphone, inteligencia emocional, y diferentes indicadores de malestar psicológico con los niveles de nomofobia, así como examinar la capacidad predictiva de estos factores para explicar esta fobia en una muestra de adolescentes. Los resultados obtenidos parecen sugerir que ciertas dimensiones de estos factores eran predictores significativos de los niveles de nomofobia.

En primer lugar, de forma descriptiva y preliminar, se examinaron los niveles de nomofobia informados por esta muestra de adolescentes, encontrándose que, en general, los participantes del estudio presentan un nivel moderado de nomofobia. En concreto, las dimensiones con puntuaciones más elevadas fueron el "miedo a no poder comunicarse" y "no ser capaz de acceder a la información". Este hallazgo sugiere que la nomofobia es una fobia situacional, posiblemente influenciada por el estilo de vida rápido y con acceso a información y compartir social en tiempo real. Además, estos resultados van en línea con un fenómeno de 
reciente estudio en el campo de las adicciones al smartphone, el llamado Fear of Missing Out (FoMO), que se define como el temor a que los demás estén divirtiéndose sin la presencia de uno, caracterizado por el deseo de estar continuamente conectado e informado de lo que otros amigos están haciendo (Przybylski, Murayama, DeHaan y Gladwell, 2013). Futuras investigaciones podrían examinar si estos síntomas de FoMO, podrían estar asociados con el desarrollo de la adicción al smartphone y la aparición de trastornos fóbicos como la nomofobia y el papel de las habilidades emocionales en el manejo de estas ansiedades de carácter tecnológico.

Por otra parte, se constataron evidencias empíricas de las relaciones entre diferentes factores predictores y los niveles en nomofobia. En concreto, se encontró una relación negativa y significativa entre los factores "uso de las emociones" y "regulación emocional", dimensiones de la inteligencia emocional, con los niveles de nomofobia, de forma que a mayores niveles de estas habilidades emocionales menores eran los niveles informados de nomofobia. Estudios previos han confirmado el papel de los déficits emocionales como un importante constructo asociado a los trastornos fóbicos y de ansiedad (Jacobs et al., 2008; Lizeretti y Extremera, 2011). Nuestros hallazgos expanden estos resultados previos en fobias sociales y ponen de manifiesto que los déficits emocionales están asociados también a otros tipos de fobias, de carácter tecnológico. La asociación entre estas dimensiones emocionales y la nomofobia sugiere que una persona con altos niveles de uso de sus emociones para conseguir objetivos y metas y con capacidad para manejar sus propias emociones podrían tener mayor capacidad de controlar el tiempo del uso del smartphone, así como gestionar los impulsos relativos al miedo de no estar con su dispositivo móvil, siendo menos probable que desarrolle síntomas nomofóbicos.

Por otro lado, también se encontró una relación positiva y significativa entre las distintas dimensiones de malestar psicológico (depresión, ansiedad, estrés) y los niveles de nomofobia, en línea con investigaciones previas en el área de la adicción al smartphone (Cheever et al. 2014; Thomée et al., 2011). Nuestros hallazgos ponen de relieve la relación fuertemente constatada entre los niveles bajos de salud mental y la aparición de síntomas fóbicos, sugiriendo la comorbilidad existente entre ambos constructos.

Además, nuestros resultados confirmaron la asociación positiva y significativa entre la adicción al smartphone y nomofobia. Así, niveles altos de adicción al smartphone se asociaban positiva y significativamente con mayores niveles de nomofobia. De acuerdo con investigaciones previas, la adicción al smartphone previa es considerado uno de los factores más predictivos en el inicio, desarrollo y mantenimiento de los síntomas nomofóbicos (González et al. 2017; Yıldız, 2018).

Respecto al último objetivo, nuestros resultados ponen de relieve el papel predictor de dos dimensiones de inteligencia emocional, los niveles de ansiedad informados y, especialmente, adicción al smartphone previa sobre sobre los niveles de nomofobia. De manera más específica, personas con alta percepción interpersonal, y por tanto, demasiado pendientes a si la necesitan o a lo que les ocurre a las personas de su entorno; con baja asimilación emocional, es decir, poco automotivadas, con elevada ansiedad y niveles altos de adicción al smartphone, son más proclives al desarrollo de trastornos nomofóbicos, siendo conveniente atender a adolescentes con dicho perfil para procurar una prevención que les permita reducir el riesgo a desarrollar nomofobia

\section{Conclusión y limitaciones}

Nuestros resultados indican el papel predictivos de ciertos déficits emocionales y desajustes psicológicos como posibles precursores de miedos irracionales de carácter tecnológico tales como la nomofobia. Estos hallazgos podrían proporcionar una guía práctica para que los centros educativos y agentes activos de aprendizaje (profesores y familias) intervengan sobre la prevalencia de la nomofobia, percibida como una de las grandes problemáticas sociales crecientes del siglo XXI (King et al., 2010; Yildirim, 2014).

No obstante, es conveniente remarcar algunas de las limitaciones del presente estudio. En primer lugar, debido a que se trata de un estudio con un diseño transversal, no se pueden llevar a cabo inferencias causales y, futuros trabajos deberían examinar, mediante estudios prospectivos longitudinales, la dirección causa-efecto entre las variables objeto de estudio. Del mismo modo, el presente estudio se desarrolla desde un enfoque cuantitativo, por lo que se recomienda, para futuras investigaciones, estudios de carácter cualitativo o con un diseño mixto, para profundizar en los problemas que las personas con nomofobia experimentan, así como para evitar que los resultados puedan estar sesgados por aspectos como la deseabilidad social, presente en la metodología basada en cuestionarios. Por otra parte, puesto que la investigación se realizó bajo un muestreo intencional no probabilístico, no es posible realizar generalizaciones, por ello, se requiere futuros trabajos con muestra más heterogénea y representativas.

\section{Implicaciones y futuras líneas}

Como futuras líneas de investigación, además de la realización de estudios longitudinales, con mayor población o con un enfoque cualitativo o mixto, también se podría ampliar la perspectiva de la presente investigación, considerando otras variables psicosociales como podrían ser el Fear of Missing Out (FoMO), debido a que el miedo a no poder comunicarse y el no ser capaz de acceder a la información, fueron los factores más destacados en los niveles de nomofobia; o la adicción a Internet, ya que la mayoría $(92,6 \%)$ de la población española, accede a Internet mediante su smartphone (INE, 2018). Respecto a variables sociodemográficas, también se podría comprobar si el desempeño académico se asocia con los niveles de nomofobia. En este sentido, Cheever, Rosen, Carrier y Chavez (2014), indican que estudiantes que informan niveles elevados de nomofobia presentan problemas de concentración o alteración de sus procesos cognitivos básicos (memoria, atención, capacidad de razonamiento). Tal como señalan Samaha y Hawi (2016) y Yıldız (2018), parece evidente que estos síntomas afectan negativamente el desempeño académico de los estudiantes.

En cuanto a las implicaciones prácticas, resulta importante atender al uso razonable de la tecnología tanto en el ámbito académico como familiar, ya que puede perjudicar la salud mental e, incluso, el desempeño académico de los estudiantes. Por lo que se proponen intervenciones en colegios e institutos que eduquen al profesorado sobre los beneficios y riesgos que tiene la tecnología en el ámbito pedagógico, qué límites se deben considerar en el uso de esa tecnología y cómo ayudar que los estudiantes utilicen de una manera consciente y respetuosa sus dispositivos móviles, poniendo especial énfasis en quienes presenten ansiedad o adicción al smartphone.

Además, también se proponen intervenciones en la prevención de la adicción a las nuevas tecnológicas y los fenómenos de riesgos asociados tales como la nomofobia. Por un lado, realizando talleres con el alumnado para ofrecerles pautas de uso razonable de los dispositivos móviles; y por otro, con las familias para ofrecerles información sobre los perjuicios que dicha adicción conlleva, así como para elaborar, de manera conjunta, pautas de mediación parental para un uso racional de dichos dispositivos, pudiendo evitar o reducir problemáticas relacionadas a la adicción al smartphone, la nomofobia u otros fenómenos emergentes como son el cyberbullying, sexting o grooming.

Por otra parte, resultaría beneficioso el desarrollo de programas enfocados en la enseñanza de las habilidades emocionales y la reducción de la ansiedad, destacando que las habilidades emocionales nos permiten una mejor gestión de las emociones negativas asociadas al abuso de la tecnología. Además, los deficits en inteligencia emocional predisponen a las personas a desarrollar mayor preferencia por las interacciones sociales online, lo cual incrementa el riesgo de adicción al smartphone (Casale, Tella, y Fioravanti, 
2013). Así pues, los programas de desarrollo de la IE ayudarían a mejorar las relaciones interpersonales de los adolescentes y potenciar un funcionamiento social más positivo, incrementándose las oportunidades para sus interacciones y un marco de comunicación más óptimo con iguales y con menos riesgos tecnológicos.

\section{Referencias}

Amichai, Y., Wainapel, G., y Fox, S. (2002). "On the Internet no one knows I'm an introvert": Extroversion, neuroticism, and Internet interaction.CyberPsychology \& Behavior, 5, 125-128. https:// doi.org/10.1089/109493102753770507

Argumosa, L., Boada, J. y Vigil-, A. (2017). Exploratory investigation of theoretical predictors of nomophobia using the Mobile Phone Involvement Questionnaire (MPIQ). Journal of Adolescence, 56, 127-135. https://doi.org/10.1016/j.adolescence.2017.02.003

Bados, A., Solanas, A. y Andrés, R. (2005). Psychometric properties of the Spanish version of Depression, Anxiety and Stress Scales (DASS). Psicothema, 17, 679-683.

Bhattacharya, S., Bashar, M. A., Srivastava, A. y Singh, A. (2019). Nomophobia: NO MObilePHonePhoBIA. Journal of Family Medicine and Primary, 8, 1297-1300. https://doi.org/10.4103/ jfmpc.jfmpc_71_19

Bragazzi, N. L. y Del Puente, G. (2014). A proposal for including nomophobia in the new DSM-V. Psychology Research and Behavior Management, 7, 155-160. https://doi.org/10.2147/PRBM.S41386

Byun, S., Ruffini, C., Mills, J.E., Douglas, A.C., Niang, M., Stepchenkova, S. y Blanton, M. (2009). Internet Addiction: Metasynthesis of 1996 -2006 Quantitative Research. CyberPsychology \& Behavior, 12(2), 203-207. https://doi.org/10.1089/cpb.2008.0102

Casale, S., Tella, L., y Fioravanti, G. (2013). Preference for online social interactions among young people: Direct and indirect effects of emotional intelligence. Personality and Individual Differences, 54, 524-529, https://doi.org/10.1016/j.paid.2012.10.023

Cheever, N. A., Rosen, L. D., Carrier, L. M. y Chavez, A. (2014). Out of sight is not out of mind: The impact of restricting wireless mobile device use on anxiety levels among low, moderate and high users. Computers in Human Behavior, 37, 290-297. https:// doi.org/10.1016/j.chb.2014.05.002

Chiu, S. I. (2014). The relationship between life stress and smartphone addiction on taiwanese university student: A mediation model of learning self-efficacy and social self-efficacy. Computers in $\mathrm{Hu}$ man Behavior, 34, 49-57. Doi: https://doi.org/10.1016/ j.chb.2014.01.024

Cho, S., y Lee, E., (2016). Distraction by smartphone use during clinical practice and opinions about smartphone restriction policies: a cross -sectional descriptive study of nursing students. Nurse Education Today, 40, 128-133. https://doi.org/10.1016/j.nedt.2016.02.021

Chotpitayasunondh, V. y Douglas, K. M. (2016). How phubbing becomes the norm: The antecedents and consequences of snubbing via smartphone. Computers in Human Behavior, 63, 9-18. https:// doi.org/10.1016/j.chb.2016.05.018

Choy, Y., Fyer, A. J., y Lipsitz, J. D. (2007). Treatment of specific phobia in adults. Clinical Psychology Review, 27(3), 266-286. https:// doi.org/10.1016/j.cpr.2006.10.002

Dixit S., Shukla H., Bhagwat A., Bindal A., Goyal A., Zaidi A.K. y Shrivastava A. (2010) A study to evaluate mobile phone dependence among students of a medical college and associated hospital of central India. Indian Journal of Community Medicine, 35(2), 339-341. https://doi.org/10.4103/0970-0218.66878

Essau, C., Conradt, J. y Petermann, F. (1999). Frequency and comorbidity of social phobia and social fears in adolescents. Behaviour Research and therapy, 37, 831-843. https:// doi.org/10.1016/S0005-7967(98)00179-X

Extremera, N., Rey, L. y Sánchez, N. (2019). Validation of the Spanish version of de Wong Law Emotional Intelligence Scale (WLEIS). Psichothema, 31(1), 94-100. https:// doi.org/10.7334/psicothema2018.147

Forgays, D. K., Hyman, I., y Schreiber, J. (2014). Texting everywhere for everything: Gender and age differences in cell phone etiquette and use. Computers in Human Behavior, 31, 314-321. https://doi.org/10.1016/j.chb.2013.10.053

Fundación Telefónica (2016). La sociedad de la información en España 2015. España: Unigraf, S.L. Recuperado de file://C:/Users/ neryelith/Downloads/

LaSociedaddelaInformacionenEspana2015.pdf

González, J., León, A., Pérez, C. y Calvete, E. (2017). Adaptación al español del cuestionario Nomophobia Questionnaire (NMP-Q) en una muestra de adolescentes. Actas Españolas de Psiquiatría, 45(4), 137-44.

Instituto Nacional de Estadística. Encuesta sobre Equipamiento y Uso de Tecnologías de Información y Comunicación en los Hogares, 2018. España. Recuperado de https://www.ine.es/prensa/ tich_2018.pdf

Jacobs, M., Snow, J., Geraci, M., Vythilingam, M., Blair, R. J. R., Charney, D. S., et al. (2008). Association between level of emotional intelligence and severity of anxiety in generalized social phobia. Journal of Anxiety Disorders, 22, 1487-1495. https://doi.org/10.1016/j.janxdis.2008.03.003

Jeong, S. H., Kim, H., Yum, J. Y. y Hwang, Y. (2016). What type of content are smartphone users addicted to?: SNS vs. games. Computers in Human Behavior, 54, 10-17. https:// doi.org/10.1016/j.chb.2015.07.035

Kang, S., y Jung, J. (2014). Mobile communication for human needs: A comparison of smartphone use between the US and Korea. Computers in Human Behavior, 35, 376-387. https:// doi.org/10.1016/j.chb.2014.03.024

Kardefelt, D. (2014). A conceptual and methodological critique of internet addiction research: Towards a model of compensatory internet use. Computers in Human Behavior, 31, 351354.https://doi.org/10.1016/j.chb.2013.10.059

King, A. L. S., Valença, A. M., y Nardi, A. E. (2010). Nomophobia: The mobile phone in panic disorder with agoraphobia: Reducing phobias or worsening of dependence? Cognitive and Behavioral Neurology, 23(1), 52-54. https://doi.org/10.1097/ WNN.0b013e3181b7eabc

King, A. L. S., Valença, A. M., Silva, A. C., Sancassiani, F., Machado, S., y Nardi, A. E. (2014). "Nomophobia'?: Impact of cell phone use interfering with symptoms and emotions of individuals with panic disorder compared with a control group. Clinical Practice and Epidemiology in Mental Health, 10, 28 35. https://doi.org/10.2174/1745017901410010028

Kwon, M., Lee, J. Y., Won, W.Y., Park, J. W., Min, J. A. y Kim, D. -J. (2013). Development and validation of a Smartphone Addiction Scale (SAS). PloS One, 8(2), 56936. https:// 
doi.org/10.1371/journal.pone.0056936

Lee, S., Tam, C. L., y Chie, Q. T. (2013). Mobile phone usage preferences: The contributing factors of personality, social anxiety and loneliness. Social Indicators Research, 118(3), 12051228. https://doi.org/10.1007/s11205-013-0460-2

Lee, Y. K., Chang, C. T., Lin, Y. y Cheng, Z. H. (2014). The dark side of smartphone usage: Psychological traits, compulsive behavior and technostress. Computers in Human Behavior, 31, 373 -383. https://doi.org/10.1016/j.chb.2013.10.047

Lizeretti, N. P., \& Extremera, N. (2011). Emotional intelligence and clinical symptoms in outpatients with generalized anxiety disorder (GAD). Psychiatric Quarterly, 82(3), 253-260. https://oi.org/10.1007/s11126-011-9167-1

López, O. (2015). Short version of the Smartphone Addiction Scale adapted to Spanish and French: Towards a cross-cultural research in problematic mobile phone use. Addictive Behaviors 64, 275-280. https://doi.org/10.1016/ j.addbeh.2015.11.013

Lovibond PF, Lovibond SH. Manual for the depression anxiety stress scales (2nd ed.). Psychology Foundation, Sydney. 1995. https://doi.org/10.1037/t01004-000

Mayer, J. y Salovey, P. (1997). What is emotional intelligence? Emotional development and emotional intelligence: Implications for educators. New York: Basic Books

Ohayon, M. y Schatzberg, A. (2010). Social phobia and depression: Prevalence and comorbidity. Journal of Psychosomatich Research, 68(3), 235-243. https://doi.org/10.1016/ j.jpsychores.2009.07.018

Park, N. y Lee, H. (2013). Nature of youth smartphone addiction in Korea: Diverse dimensions of smartphone use and individual traits. Journal of Communication Research, 51(1), 100-132. https://doi.org/10.22174/jcr.2014.51.1.100

Park, N., Kim, Y. C., Shon, H. Y., y Shim, H. (2013). Factors influencing smartphone use and dependency in South Korea. Computers in Human Behavior, 29(4), 1763-1770. https:// doi.org/10.1016/j.chb.2013.02.008

Przybylski, A. K., Murayama, K., DeHaan, C.D. y Gladwell, V. (2013). Motivational, emotional, and behavioral correlates of fear of missing out. Computers in Human Behavior, 29, 1841184. https://doi.org/10.1016/j.chb.2013.02.014

Salehan, M. y Negahban, A. (2013). Social networking on smartphones: When mobile phones become addictive. Computers in Human Behavior, 29(6), 2632-2639. https://doi.org/10.1016/ j.chb.2013.07.003

Samaha, M. y Hawi, N. S. (2016). Relationships among smartphone addiction, stress, academic performance, and satisfaction with life. Computers in Human Behavior, 57, 321-325. https:// doi.org/10.1016/j.chb.2015.12.045

Sánchez, N., Extremera, N. y Fernández, P. (2016). The relation between emotional intelligence and subjective well-being: A meta-analytic investigation. Journal of Positive Psychology, 11, 276- 285. https://doi.org/10.1080/17439760. 2015.1058968

Sapacz, M., Rockman, G. y Clark, J. (2016). Are we addicted to our cell phones? Computers in Human Behavior, 57, 153159.https://doi.org/10.1016/j.chb.2015.12.004
SecurEnvoy (2012). Recuperado de https://www.securenvoy.com/en-gb/ blog/66-population-suffer-nomophobia-fear-being-without-theirphone

Sezer B. y Atilgan S.B.C. (2018) The Dark Side Of Smartphone Usage (Nomophobia): Do We Need To Worry About It? TıpEğitimiDünyas1, 18(54), 30-43. https://doi.org/10.25282/ted.513988

Summerfeldt, L. J., Kloosterman, P. H., Antony, M. M., McCabe, R. E. y Parker, J. D. A. (2010). Emotional Intelligence in Social Phobia and Other Anxiety Disorders. Journal of Psychopathology Behaviour Assessment, 33, 69-78. https://doi.org/10.1007/s10862-0109199-0

Sun, J., Liu, Q., y Yu, S. (2019). Child neglect, psychological abuse and smartphone addiction among Chinese adolescents: The roles of emotional intelligence and coping style. Computers in Human Behavior, 90, 74-83. https://doi.org/10.1016/j.chb.2020.106296

Thomée, S., Härenstam, A. y Hagberg, M. (2011). Mobile phone use and stress, sleep disturbances, and symptoms of depression among young adultsa prospective cohort study. BMC public health, 11(1), 66. https://doi.org/10.1186/1471-2458-11-66.

Torre, A. y Essau, C. (2019). Symptom network connectivity in adolescents with comorbid major depressive disorder and social phobia. Journal of Affective Disorders, 255, 60-68. https://doi.org/10.1016/ j.jad.2019.05.015

Van Deursen, A. J., Bolle, C. L., Hegner, S. M. y Kommers, P. A. (2015). Modeling habitual and addictive smartphone behavior: The role of smartphone usage types, emotional intelligence, social stress, selfregulation, age, and gender. Computers in human behavior, 45, 411-420. https://doi.org/10.1016/j.chb.2014.12.039

Wersebe, H., Lieb, R., Meyer, A., Miche, M., Mikoteit, T., Imboden, C., Hoyer, J., Bader, K., Hatzinger, M., y Gloster, A. (2018). Wellbeing in major depression and social phobia with and without comorbidity. International Journal of Clinical and Health Psychology, 18(3), 201-208. https://doi.org/10.1016/j.ijchp.2018.06.004

Wong,C.S. y Law, K.S. (2002). The effects of leader and followeremotional intelligence on performance and attitude: An exploratorystudy. Leadership Quarterly, 13, 243-274. https://doi.org/10.1016/S10489843(02)00099-1

Yildirim, C. (2014). Exploring the dimensions of nomophobia: Developing and validating a questionnaire using mixed methods research. (Tesis doctoral). Disponible en Graduate Theses and Dissertations. 14005. https://lib.dr.iastate.edu/etd/14005

Yildirim, C. y Correia, A. (2015). Exploring the dimensions of nomophobia: Development and validation of a self-reported questionnaire. Computers in Human Behavior, 49, 130-137. https:// doi.org/10.1016/j.chb.2015.02.059

Yildiz, H. (2019). Investigation of nomophobia and smartphone addiction predictors among adolescents in Turkey: Demographic variables and academic performance. The Social Science Journal, 4, 492517. https://doi.org/10.1016/j.soscij.2018.09.003

Zhitomirsky, M. y Blau, M. (2016). Cross-generational analysis of predictive factors of addictive behavior in smartphone usage. Computers in human behavior, 64, 682-693. https://doi.org/10.1016/ j.chb.2016.07.061 